\title{
Channeling Contrast in Sub-Ångström Resolution High-Angle Annular Dark- Field Images of Planar Interfaces
}

\author{
Jingyue (Jimmy) Liu* and Lawrence F. Allard** \\ * Center for Nanoscience, Department of Physics and Astronomy, Department of Chemistry and \\ Biochemistry, University of Missouri-St. Louis, St. Louis, MO 63121 \\ ** Materials Science \& Tech. Division, Oak Ridge National Laboratory, Oak Ridge, TN 37831
}

Sub-Ångström resolution imaging in aberration-corrected STEM is now routinely available, providing atomic scale information on structure, composition and, in some cases, electronic states of the probed sample. Quantitative STEM, however, requires better understanding of the contrast mechanisms of STEM images. We reported earlier the effect of surface-resonance channeling on the contrast of sub-Ångström resolution high-angle annular dark-field (HAADF) images [1]. We report here our recent investigation of the effect of electron beam channeling on the contrast of HAADF images of coherent or semi-coherent planar interfaces.

The $\mathrm{ZnO}$ nanobelts used in these experiments were made by a thermal evaporation-condensation method. Each nanobelt is a single crystal with relatively uniform thickness; some nanobelts possess atomically flat and clean surfaces. Palladium atoms/clusters were chemically dispersed onto these $\mathrm{ZnO}$ nanobelts. After heating the $\mathrm{Pd} / \mathrm{ZnO}$ samples inside the electron microscope at $500^{\circ} \mathrm{C}, \mathrm{PdZn}$ alloy nanoparticles grew epitaxially on the surfaces of the nanobelts, forming coherent or semicoherent interfaces between the PdZn alloy nanoparticles and the $\mathrm{ZnO}$ nanobelts. A JEOL 2200FS FEG STEM/TEM with a hexapole aberration corrector (CEOS GmbH, Heidelberg, Ger.) for the probe-forming optics, providing a nominal resolution of $0.07 \mathrm{~nm}$ in HAADF imaging mode, was used to obtain the HAADF images.

Figure 1a shows a HAADF image of a PdZn alloy nanoparticle growing epitaxially onto the (11-20) surface of a $\mathrm{ZnO}$ nanobelt. The PdZn alloy nanoparticle has a cubo-octahedral shape and was oriented along the [001] zone axis with the (110) plane parallel to the $\mathrm{ZnO}$ (0001) plane. The $\mathrm{ZnO}$ nanobelt was tilted slightly off the [11-20] zone axis. When a PdZn nanoparticle ( $1_{0}$ structure) is oriented along the [001] zone axis, the HAADF image should reveal layers of Pd and $\mathrm{Zn}$ with good image contrast. Figure 1a, however, displays a prominent stripe contrast across the PdZn alloy nanoparticle, overshadowing the contrast of the individual $\mathrm{Pd}$ and $\mathrm{Zn}$ layers of the $\mathrm{PdZn}$ alloy. Figure $1 \mathrm{~b}$ shows the same PdZn nanoparticle obtained after an almost negligible tilt of the sample, displaying different contrast of the individual columns of atoms from that shown in Fig. 1a. Figure 2a shows another PdZn alloy nanoparticle oriented exactly along the [011] zone axis with the (11-1) plane parallel to the $\mathrm{ZnO}$ (0001) plane. The $\mathrm{ZnO}$ nanobelt was tilted to the exact [11-20] zone axis. The image contrast of the PdZn nanoparticle is dominated by 1) domains of clearly resolved columns of atoms with bright contrast, and 2) domains of highly distorted "columns of atoms" with grey contrast. Figure $2 \mathrm{~b}$ shows the same PdZn alloy nanoparticle obtained after a slight tilt of the $\mathrm{ZnO}$ nanobelt, revealing a dramatic change of the image contrast of the alloy nanoparticle. We propose that the observed contrast variations of the HAADF images discussed above are caused by the strain field at the semi-coherent interfaces. The misfit dislocation networks at the semi-coherent interphase interfaces change the local strain field which modifies the channeling condition of the sub-Ångström electron probe, resulting in contrast variations in HAADF images of planar interfaces. The channeling contrast of sub-Ångström resolution HAADF images of semi-coherent interfaces 
may provide information on the two dimensional distribution of misfit dislocations and the atomic arrangement across semi-coherent interfaces. The detailed contrast mechanisms of electron channeling across planar and edge-on interfaces will be discussed [2].

\section{References}

[1] J. Liu and L. F. Allard, Microsc. Microanal., 16 (2010) 425.

[2] This research was supported by UM-St. Louis; microscopy research at the Oak Ridge National Laboratory's High Temperature Materials Laboratory was sponsored by the U. S. Department of Energy, Office of Energy Efficiency and Renewable Energy, Vehicle Technologies Program.


FIG. 1. a) HAADF image of a PdZn alloy nanoparticle growing epitaxially on the (11-20) surface of a $\mathrm{ZnO}$ nanobelt, revealing a predominant stripe contrast across the alloy nanoparticle; b) the same alloy nanoparticle after a slight tilt from that of a). The PdZn alloy nanoparticle was oriented along the [001] zone axis and the $\mathrm{ZnO}$ nanobelt was tilted slightly off the [11-20] zone axis.

FIG. 2. a) HAADF image of a PdZn alloy nanoparticle growing epitaxially on the (11-20) surface of a $\mathrm{ZnO}$ nanobelt, revealing domains of clearly resolved columns of atoms and domains of highly distorted "columns of atoms" across the alloy nanoparticle. The PdZn alloy nanoparticle was oriented exactly along the [011] zone axis and the $\mathrm{ZnO}$ nanobelt was tilted to the exact [11-20] zone axis. b) HAADF image of the same alloy nanoparticle as that shown in a) but after a slight tilt of the $\mathrm{ZnO}$ nanobelt, revealing significant contrast change from that of a). 